\title{
Mobile Robot Localization: A Review of Probabilistic Map- Based Techniques
}

\author{
Salvador M. Malagon-Soldara, Manuel Toledano-Ayala, Genaro Soto-Zarazua, \\ Roberto V. Carrillo-Serrano, Edgar A. Rivas-Araiza \\ * Division de Estudios de Posgrado, Facultad de Ingenieria, Universidad Autonoma de Queretaro, Queretaro, Mexico
}

\begin{tabular}{l}
\hline \hline Article Info \\
\hline Article history: \\
Received Aug 8, 2014 \\
Revised Nov 12, 2014 \\
Accepted Nov 28, 2014 \\
\hline
\end{tabular}

Keyword:

Bayesian inference

Kalman filter

Localization

Mobile robot

Particle filter

\begin{abstract}
This work presents a comprehensive review of current probabilistic developments used to calculate position by mobile robots in indoor environments. In this calculation, best known as localization, it is necessary to develop most of the tasks delegated to the mobile robot. It is then crucial that the methods used for position calculations be as precise as possible, and accurately represent the location of the robot within a given environment. The research community has devoted a considerable amount of time to provide solutions for the localization problem. Several methodologies have been proposed the most common of which are based in the Bayes rule. Other methodologies include the Kalman filter and the Monte Carlo localization filter wich will be addressed in next sections. The major contribution of this review rests in offering a wide array of techniques that researchers can choose. Therefore, method-sensor combinations and their main advantages are displayed.
\end{abstract}

Copyright (C) 2015 Institute of Advanced Engineering and Science. All rights reserved.

\section{Corresponding Author:}

Edgar A. Rivas-Araiza,

Division de Estudios de Posgrado, Facultad de Ingenieria,

Universidad Autonoma de Queretaro,

Cerro de las campanas S/N, Queretaro, Mexico.

Email: erivas@uaq.mx

\section{INTRODUCTION}

Mobile robot have seen an increase presence in the industrial field performing tasks as varied as cleaning floors, loading and unloading in industrial plants, transporting samples from one laboraty to another, among many others. All that without ignoring the growing introduction of this type of robot as a domestic worker because of its flexibility, small size and low cost. Nearly all of these applications require knowledge of the position of the robot; therefore it is necessary to perform a localization calculation [1]. In localization, the position of the robot relative to a map of an environment is estimated and this calculation represents one of the most relevant problems in mobile robotics [2]. Furthermore, these calculations are used in other modules of the robot control software that are in charge of deciding how the robot should act in the next movement. [3] establish that the robot must navigate safely within its environment as a key prerequisite for a truly autonomous robot. Reliable navigation in mobile robotics requires the computation of robust motion approximations. Solutions based on inertial measurement units or global positioning system (GPS) can provide position approximations and their corresponding uncertainties [4]. However, this solution is impractical in indoor applications where GPS signals are not reliable. While outdoor localization in open areas has been largely solved with the advances in satellite-based GPS systems, indoor localization presents ongoing challenges due to the large range of variables that require different techniques [5]. As it is not possible to have a calculation using GPS, the use of other types of sensors is necessary to collect information from the environment. Two different sources of information may be used to map navigation: proprioceptive (gyroscope, inclinometer) and exteroceptive (compass). Some authors call these sensors as idiothetic and 
allothetic sensors, respectively [6]. The robot gathers data through exteroceptive sensors which survey the world and proprioceptive sensors which continuously monitor the motion of the robot in space via compass readings, wheel encoders, and others [7]. These sensors are used to determine the orientation and inclination of the robot; the process to calculate the orientation also it is called attitude estimation. At the same time, the technique of estimating the position through the initial position, course and speed, is called dead reckoning [8]. In dead reckoning (heading sensors) and odometry (wheel sensors only) the position update is based on proprioceptive sensors. The movement of the robot is sensed with wheel encoders and/or heading sensors that later it is integrated to compute position. Because the sensor measurement errors also are integrated, the position error is accumulated over time. Thus the position has to be updated from time to time by other localization mechanisms; otherwise the robot is unable to maintain a meaningful position estimate in long runs. In short, mobile robot effectors introduce uncertainty about the next state. Thence, it is important to understand the precise nature of the effector noise that affects mobile robots. From the robot perspective, this error in motion is viewed as an error in odometry, or the inability of the robot to estimate its own position over time using knowledge of its kinematics and dynamics. In the field of mobile robotics, it is common that odometry error sources be divided into two different groups. The first source is the systematic error that is deterministic. Systematic error sources include unequal wheel diameters, misalignment of wheels, or kinematic modeling errors. Therefore, it is possible to decrease the error if kinematic parameters are calibrated. The second source is the nonsystematic error, which is stochastic. Possible sources of these kinds of errors are environmental conditions such as uneven ground or wheel slippage. Nonsystematic errors cannot be directly compensated, but the errors are just modeled as the stochastic uncertainty. If nonsystematic errors are too large, then it is difficult to use pure odometry for position estimation [9].

The true source of error generally lies in an incomplete model of the environment, which represent a nonsystematic error. For instance, the robot does not model the fact that the floor may be sloped, the wheels may slip, or that a human may push the robot. All of these unmodeled sources of error result in inaccuracy between the physical motion of the robot, the intended motion of the robot, and the proprioceptive sensor estimates of motion [10]. Furthermore, interaction between the robot and the environment, along with the presence of noisy sensor readings make the problem more difficult to solve. Another type of problem occurs when the measurements of sensors arrive delayed to the localization module due to multiple factors such as the physical distribution of the sensors, the communication network, and the time used to pre-process the raw measurements to extract the information that is sent to the localization module. Another difficult scenario appears when the delays and the sequence of the arrival of information to the localization module are not fixed, constituting the out-of-sequence problem (OOSP). In order to deal with the measurement arrival delays, the localization module can basically implement four different solutions, as suggested in [11].

Briefly, the autonomous mobile robot starts from an initial position without prior knowledge of the environment and tries to gain information about its surroundings, through its onboard sensor measurements. The robot needs to consider all of the measurements from the sensors to create a belief of its next state. In this order to achieve this it is necessary employ a probabilistic method. Here, the classical Bayesian formulation is adopted to update a hypothesis. Hence, sensor measurements are combined to calculate the location of salient features of the environment (mapping process) and simultaneously the robot estimates its own position in this continuously enriched map (localization process). In general, the majority of works in the literature relies on probabilistic frameworks to solve the localization problem. The idea underpinning such approaches is to recursively maintain a probability distribution, called belief, over all positions (state space points) in the environment. Probabilistic localization algorithms are variants of the Bayes filter. The straightforward application of Bayes filters to the localization problem is called Markov localization. The Markov localization model can represent any probability density function regarding robot position. However, this approach is extremely general and some authors describe it as inefficient. Considering the fundamental demands on a robot localization system, one can argue that this filter is not the correct solution to the localization problem but sensor fusing is a key element to robust localization. The Kalman filter is presented in the next section. This method is commonly applied to satisfactorily combine sensor measurements followed by an analysis of other algorithms derived from the Bayes rule. In this work, frameworks with the same sensor type used are divided in different sections, and accordingly it is possible compare the features contained in similar systems.

\section{KALMAN FILTER}

To control a mobile robot, as explained above, frequently it is necessary to combine information from multiple sources. However, different types of sensors have different resolutions and degrees of error. Consequently, the information that comes from trustworthy sources should be more important or carry more weight than less reliable ones. A general way to compute the information from sources that are more or less 
trustworthy and what weights must be given to the data of each source; is by making a weighed pounder addition of the measurements. This process is better known as Kalman filter and it is one of the methods more widely used for sensorial fusion in mobile robotics applications [12]. In Figure 1, a Kalman filter is illustrated where the blocks represent the measurements, devices, and the environment. This filter is used when the system to be modeled fails for having a nonlinear Gaussian noise distribution. While the errors are approximately Gaussian, the Kalman filter can be used nevertheless but will probably not be optimal. For nonlinear systems, the extended Kalman filter (EKF) is used. This involves the linearization of the plant, and if necessary, the linearization of the measurement. Thus, high order terms of the Taylor expansion are cancelled. The existing linearized error propagation in the family of Kalman filters can result in large errors and inconsistency in the simultaneous localization and mapping (SLAM) problem. One approach to alleviate this situation is the use of iteration in the EKF and the sigma point Kalman filter (SPKF) [13].

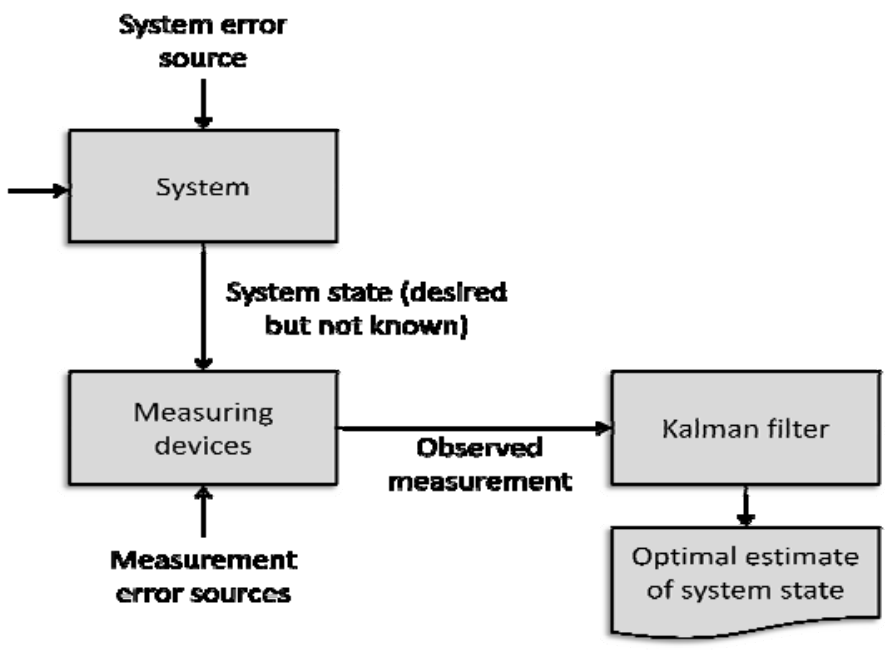

Figure 1. Typical Kalman filter application

To review the work done by the scientific community, this section is divided into two different types of frameworks. First, works that use landmarks are displayed. The majority of these works include vision sensors and triangulation methods. And second, methods based on laser sensors are shown. These two frameworks represent the effort to improve the solution to the localization problem, and their importance is highlighted individually.

\subsection{Landmark and triangulation methods}

As navigation strategy, methods with landmarks and triangulation of signals rely on identification of features or objects of the environment. The features and objects must be known a priori or extracted dynamically. The environment features are divided into four types: 1) active beacons that are fixed at known positions and actively transmit ultrasonic, IR (infrared) or RF (radio frequency) signal for the calculation of the absolute robot position through the direction of receiving incidence; 2) artificial landmark which are specially designed objects or markers placed at known locations in the environment; 3) natural landmarks which are distinctive environmental features and can be extracted by sensors; and 4) environment models that are built from prior knowledge about the environment and can be used for matching new sensor observations. Among the environmental features discussed, natural landmark-based navigation is flexible because no explicit artificial landmarks are needed, but may not work well when landmarks are sparse or the environment is not known a priori. Although the artificial landmarks are enhanced and map building process is simplified. Nowadays, the emergence of visual sensors has resulted in a trend towards the use of digital cameras as the main sensor to gather information. The simultaneous process of localization and mapping through cameras is commonly called visual SLAM and solved with EKF. The basic strength of EKF in solving the SLAM problem lies in its iterative approach of determining the estimation. Henceforth building of an augmented map of its surrounding environment where the robots navigate through some waypoints. [14] gradually build the map by considering it as an augmentation of estimated states, which are nothing but a collection of positions of the features (or landmarks) in the environment, along with the position of the robot. Thus, to solve the localization problem, the robot position and the locations of observed stationary 
landmarks (for example line segments) must be estimated. The observation-update step requires that all the landmarks and the joint covariance matrix be updated every time an observation is made. This means that the extent of the computation expands quadratically with the number of landmarks in a map. Besides, visionbased approaches present several problems with occlusions, real-time operation, and environment modifications. Consequently, the robot can only detect the presence of the tags when it is traveling in their proximity. As a result, the importance of combining this information with data obtained from other sensors (e.g. odometry) is observed [15] [16]. Below a laser range finder is shown. This type of sensor facilitates the data processing by the localization algorithm. Besides, its recognition does not depend on changes in the environment.

\subsection{Laser range finder}

The localization system based on the laser scanner and retro-reflective landmarks is a promising absolute positioning technique in terms of performance and cost. The laser actively emits a signal and records its echo, the output signal being a light beam. Lasers provide much more focused beams than other sensors like sonars. This is crucial when hitting a smooth surface at an angle. [17] use sensor fusion between an omnidirectional camera and a 3D laser range finder (LRF). This approach takes advantage of the metric information provided by the LRF and combines it with the omnidirectional vision. Then camera extracts the vertical lines in the environment and using a scan matching technique, solves the SLAM problem. However, the authors do not consider occlusions and illumination changes.

In [18], the EKF is used to localize the mobile robot with a LRF sensor in an environment demarcated with line segments. Simulating the kinematic model of the robot performs a prediction step. A method for estimating the covariances of the line parameters based on classic least squares (LSQ) is proposed. This method is compared with the method resulting from the orthogonal LSQ in terms of computational complexity. The results of a comparison show that the use of classic LSQ instead of orthogonal LSQ reduces the number of computations in a localization algorithm that is a part of a SLAM. In the input noise covariance matrix of the EKF the standard deviation of each angular speed of robot wheels is calculated as being proportional to the angular speed of the robot wheels. A correction step is performed minimizing the difference between the matched line segments from the local and global maps [19]. If the overlapping rate between the most similar local and global line segments is below the threshold, the line segments are paired. The line covariances of parameters, which arise from the LRF distance-measurement error, comprise the output noise covariance matrix of the EKF. Line segments were chosen because they require a smaller amount of computer memory in comparison with the occupancy grids method [20].

Traditionally, many nonlinear Bayesian estimation problems are solved using the EKF. But when the dynamic models and measurements are highly nonlinear and the measurement noise is not Gaussian, linearized methods may not always be a good approach [21]. Popular alternatives to Gaussian techniques are nonparametric filters. Nonparametric filters do not rely on a fixed functional form of the posterior, such as Gaussians. Instead, they approximate posteriors by a finite number of values, each roughly corresponding to a region in state space.

\section{PARTICLE FILTER}

Particle filters (PF) are sequential Monte Carlo methods under the Bayesian estimation framework and have been widely used in many fields such as signal processing, target tracking, mobile robot localization, image processing, and various economics applications. The key idea is to represent the next probability density function (PDF) of the state variables by a set of random samples or particles with associated weights, and compute estimates based on these samples and weights. PF can estimate the system states sufficiently when the number of particles (estimations of the state vectors which evolve in parallel) is large. The PF can be applied to any state transition or measurement model, and it does not matter if some errors in inertial sensors exhibit complex stochastic characteristics. These errors are hard to model using a linear estimator such as the Kalman filter because of their high inherent nonlinearity and randomness. However, this method has not yet become popular in the industry because implementation details are missing in the available research literature, and because its computational complexity has to be handled in real-time applications. The first method discussed it is the triangulation by WiFi (IEEE 802.11 WLAN), which consists in identifying access points in the environment. One advantage in using WiFi technology is its frequent use in indoor environments.

\subsection{WiFi}

According to WiFi-alliance, over 700 million people use WiFi and there are about 800 million new WiFi devices every year. This freely available wireless infrastructure prompted many researchers to develop 
WiFi-based positioning systems for indoor environments. Three main approaches for WiFi-based positioning exist: time-based, angle-based, and signal-strength-based approaches.

Often times, however, there are no available WiFi access points and it is necessary to find a new manner of identifying the environment. Omnidirectional cameras represent a cheap solution and many features of the environment can be extracted from an image.

\subsection{Omnidirectional cameras and laser range finder}

According to [22] two methodologies have been prevalent in live motion and structure estimation from a single moving video camera: i) filtering approaches that fuse measurements from all images sequentially by updating probability distributions over features and camera parameters; and ii) bundle adjustment (BA) methods that perform batch optimization over selected images from the live stream, such as sliding window, or in particular spatially distributed keyframes. In mentioned work probability distributions are studied, and accordingly to localizace the robot it is important have object recognition. Authors like [23] build a probabilistic object recognition. In proposed framework, a static recognition module that provides class propabilities for each pixel of a set of local RGB features. For this purpose two methods are presented: i) a Bayesian method based on a maximum likelihood; and ii) a neural network that by author results, it is demonstrate sometimes work better than the Bayesian approach when they are integrated within a tracking framework. [24], [25], [26] studied the feasibility of the techniques based on the global appearance of a set of omnidirectional images captured by a camera to solve the localization problem. First, they studied how to describe the visual information globally so that it correctly represents locations and the geometrical relationships between these locations. Then they integrated this information using an approach based on a spring-mass-damper model, to create a topological map of the environment. Once the map is built, they proposed the use of a Monte Carlo localization approach to estimate the most probable position of the vision system and its trajectory within the map.

[27] present a methodology to build incremental topological maps. They used omnidirectional images both in robot mapping and localization. These solutions can be categorized into two main groups: feature-based and appearance-based solutions. In the first approach, a number of significant points or regions are extracted from each omnidirectional image and each point is described using an invariant descriptor. All the experiments have been carried out with a set of omnidirectional images captured by a catadioptric system mounted on the mobile platform. Each scene is first filtered to avoid lighting dependence and then is described through a Fourier-based signature that presents a good performance in terms of amount of memory and processing time. In that work, the authors have evaluated the influence of the descriptor in the localization by varying the number of possible associations. The system is able to estimate the position of the robot in the case of an unknown initial position and it is able to track the position of the robot while moving. In the evaluated methods, as they increase the number of particles in the system, the average of localization decreases rapidly. Also, it is possible to correct the weighting of the particles by combining a physical system of forces with a Gaussian weight.

Approaches before the present, do not represent all the techniques used in the visual framework. There exist other methods with more than a camera like stereo vision. [28] solve the SLAM problem with an observation model that consider both the 3D positions and the SIFT descriptor of the lankmarks. One advantage of stereo vision is the measure of the depth and therefore the possibility of realice a probabilistic model for visual odometry. In the next section a compilation of parallel techniques is presented, many of which are focused on reducing the number of the computations.

\section{OTHER METHODS}

[29] establishes that the time and memory requirements of the basic EKF-SLAM approach result from the cost of maintaining the full covariance matrix, which is $O(n 2)$ where $n$ is the number of features in the map. Many recent efforts have concentrated on reducing the computational complexity of SLAM in large environments. Several current methods address the computational complexity problem by working on a limited region of the map. Postponement and the compressed filter significantly reduce the computational cost without sacrificing precision, although they require an $O(n 2)$ step on the total number of landmarks to obtain the full map. The split covariance intersection method limits the computational burden but sacrifices precision: it obtains a conservative estimate. The sparse extended information filter is able to obtain an approximate map in constant time per step, except during loop closing. All cited methods work on a single absolute map representation, and confront divergence due to nonlinearities as uncertainty increases when mapping large areas. In contrast, local map joining and the constrained local submap filter, propose to build stochastic maps relative to a local reference, guaranteed to be statistically independent. By limiting the size of the local map, this operation is the constant time per step. Local maps are joined periodically into a global 
absolute map, in a $O(n 2)$ step. Given that most of the updates are carried out on a local map, these techniques also reduce the harmful effects of linearization. To avoid the $O(n 2)$ step, the constrained relative submap filter proposes to maintain the independent local map structure. Each map contains links to other neighboring maps, forming a tree structure (where loops cannot be represented). In the Atlas framework [30], network coupled feature maps, and constant time SLAM the links between local maps form an adjacency graph. These techniques do not impose loop consistency in the graph thus sacrificing the optimality of the resulting global map. Hierarchical SLAM proposes a linear time technique to impose loop consistency, obtaining a close to optimal global map. The FastSLAM technique uses particle filters to estimate the vehicle trajectory and each one has an associated set of independent EKF to estimate the location of each feature in the map. This partition of SLAM into a localization and a mapping problem, allows to obtain a computational complexity $O(\log (n))$ with the number of features in the map. However, its complexity is linear with the number of particles used. The scaling of the number of particles needed with the size and complexity of the environment remains unclear. In particular, closing loops causes dramatic particle extinctions that map results in optimistic (i.e., inconsistent) uncertainty estimations.

In [31], researchers investigate the potential to improve the nonquantized (NQ) method, by exploiting the entropy-discriminativity relation. In this work they investigate the nonquantized representation as a solution to the global localization problem. In particular they focus on performance gains this representation offers over the BoW (Bag of Words) model and of the potential to improve efficiency and memory size at a reduced accuracy loss. As a first contribution, this paper presents a comparative evaluation of quantized (Q) and nonquantized representations in a robot localization task. As a second contribution, they propose modulating the importance of features according to the entropy measure, which is experimentally shown to benefit localization accuracy. As a third contribution, it proposes two approaches to speed up the NQ method at run time. In the first approach, they propose a hierarchical localization scheme performed at two stages is proposed. In the second approach, objective was to capitalize on the specificities of the training data for localization. Experimental results obtained with this method support its superiority in the global localization task and suggest that performance gains can be achieved in the loop closure problem.

Vallet [32] present a mobile robot used to simultaneously locate the nodes of a wireless network and calibrate the parameters of received signal strength. They assume that the position of all the nodes is unknown and use a mobile robot, capable of SLAM, as a mobile beacon. While the robot moves around, it builds a map of the environment using a laser scanner and odometry information. Thus, its position within the map is known at any moment. As the robot moves, it also collects RSS (received signal strength) measurements from the nodes of the network. All this information is then exploited to estimate the position of the nodes. The efficacy of the models can be compared using the likelihood of the data. However, the authors also consider that a more meaningful comparitive measure in the context of this research is to use the real error of maximum likelihood (ML) position estimates. One advantage of the ML formulation of the localization problem is that it does not require calculating the inverse model of the RSS-distance, which can be difficult. In particular, if the RSS-distance mapping is not bijective, the inverse model (distance-RSS) might contain several distances for the same RSS values. This can be a serious drawback for some algorithms that require a direct estimate of the distance from RSS, and requires additional work to choose between the possible alternatives. The ML formulation of the problem simply does not suffer from this problem, and it can work with any function of the distance, as long as the model is a valid PDF. To learn more about WiFi signal strength sensors, read [33], [34], [35], [36].

\section{DISCUSSION}

There are studies which compares the effectiveness of EKF and PF such as [37], where the EKF has been employed for the localization of an autonomous vehicle by fusing data coming from different sensors. In the EKF approach the state vector is approximated by a Gaussian random variable, which is then propagated analytically through the first order linearization of the nonlinear system. The series approximation in the EKF algorithm can, however, lead to poor representations of the nonlinear functions and of the associated probability distributions. As a result, sometimes the filter will be divergent. Related work has shown that the particle filter is superior than the EKF in terms of the accuracy of the state vector estimation, as well as in terms of robustness and tolerance to measurement noise. The performance of the particle filter algorithm depends on the number of particles and their initialization. It can be seen that the PF algorithms generate better estimates of the state vector of the mobile robot as the number of particles increases, but at the expense of higher computational effort.

The optimal filter for a linear model with Gaussian noise is the Kalman filter. State estimation for nonlinear systems with non-Gaussian noise is a difficult problem and in general the optimal solution cannot be expressed in closed-form. In order to increase the accuracy of visual SLAM it is usually more profitable to 
increase the number of features than the number of frames. This is the key reason why BA is more efficient than filtering for visual SLAM. On the other hand, the PF suffers from the so-called sample impoverishment problem in which samples tend to converge to a confined region in the solution space, making state estimations trapped in local optima. In [38], samples of particles are updated and propagated by implementing a sequential importance sampling (SIS) process recursively as new measurement information becomes available. As the number of samples becomes very large and approaches infinity, the SIS particle filter approximates the optimal Bayesian estimate.

\section{ACKNOWLEDGEMENTS}

The authors want to thank for their finantial support to:

- Consejo Nacional de Ciencia y Tecnolgia, Mexico.

- $\quad$ FOFI, Universidad Autonoma de Queretaro, Mexico.

\section{REFERENCES}

[1] C. Gamallo, et al., "Omnivision-based kld-Monte Carlo localization," Robotics and Autonomous Systems, vol. 58(3), pp. 295-305, 2010.

[2] A. Gasparri, et al., "Monte carlo filter in mobile robotics localization: a clustered evolutionary point of view," Journal of Intelligent and Robotic Systems, vol. 47(2), pp. 155-174, 2006.

[3] M.-H. Li, et al., "Novel indoor mobile robot navigation using monocular vision," Engineering Applications of Artificial Intelligence, vol. 21(3), pp. 485-497, 2008.

[4] Z. Sun, et al., "Inferring laser-scan matching uncertainty with conditional random fields," Robotics and Autonomous Systems, vol. 60(1), pp. 83-94, 2012.

[5] M. D'Souza, et al., "Evaluation of realtime people tracking for indoor environments using ubiquitous motion sensors and limited wireless network infrastructure," Pervasive and Mobile Computing, vol. 9(4), pp. 498-515, 2013.

[6] D. Filliat, et al., "Map-based navigation in mobile robots:: I. a review of localization strategies," Cognitive Systems Research, vol. 4(4), pp. 243-282, 2003.

[7] Y. Elor, et al., "A "thermodynamic" approach to multi-robot cooperative localization," Theoretical Computer Science, vol. 457, pp. 59-75, 2012.

[8] M. Ahmadi, et al., "Attitude estimation by divided difference filter in quaternion space," Acta Astronautica, vol. 75, pp. 95-107, 2012.

[9] K. Lee, et al., "Kinematic parameter calibration of a car-like mobile robot to improve odometry accuracy," Mechatronics, vol. 20(5), pp. 582-595, 2010.

[10] R. Siegwart, et al., "Introduction to autonomous mobile robots: MIT press; 2011.

[11] E. Besada-Portas, et al., "Localization of Non-Linearly Modeled Autonomous Mobile Robots Using Out-ofSequence Measurements," Sensors, vol. 12(3), pp. 2487-2518, 2012.

[12] L. F. de Melo, et al., "Trajectory planning for nonholonomic mobile robot using extended Kalman filter," Mathematical Problems in Engineering, vol. 2010, pp., 2011.

[13] K. Shojaei, et al., "Experimental Study of Iterated Kalman Filters for Simultaneous Localization and Mapping of Autonomous Mobile Robots," Journal of Intelligent \& Robotic Systems, vol. 63(3-4), pp. 575-594, 2011.

[14] A. Chatterjee, et al., "Development of a real-life EKF based SLAM system for mobile robots employing vision sensing," Expert Systems with Applications, vol. 38(7), pp. 8266-8274, 2011.

[15] M. Boccadoro, et al., "Constrained and quantized Kalman filtering for an RFID robot localization problem," Autonomous Robots, vol. 29(3-4), pp. 235-251, 2010.

[16] K. Yu, et al., "Localization algorithm using a virtual label for a mobile robot in indoor and outdoor environments," Artificial Life and Robotics, vol. 16(3), pp. 361-365, 2011.

[17] G. Gallegos, et al., "Indoor SLAM based on composite sensor mixing laser scans and omnidirectional images," in Robotics and Automation (ICRA), 2010 IEEE International Conference on; 2010: IEEE, Year, pp. 3519-3524.

[18] L. Teslić, et al., "Using a LRF sensor in the Kalman-filtering-based localization of a mobile robot," ISA transactions, vol. 49(1), pp. 145-153, 2010.

[19] A. Llarena, et al., "Odometry-Based Viterbi Localization with Artificial Neural Networks and Laser Range Finders for Mobile Robots," Journal of Intelligent \& Robotic Systems, vol. 66(1-2), pp. 75-109, 2012.

[20] L. Teslić, et al., "EKF-based localization of a wheeled mobile robot in structured environments," Journal of Intelligent \& Robotic Systems, vol. 62(2), pp. 187-203, 2011.

[21] P. Axelsson, et al., "Bayesian state estimation of a flexible industrial robot," Control Engineering Practice, vol. 20(11), pp. 1220-1228, 2012.

[22] H. Strasdat, et al., "Visual SLAM: why filter?," Image and Vision Computing, vol. 30(2), pp. 65-77, 2012.

[23] F. Serratosa, et al., "A probabilistic integrated object recognition and tracking framework," Expert Systems with Applications, vol. 39(8), pp. 7302-7318, 2012.

[24] Z. Chen, et al., "Visual detection of lintel-occluded doors by integrating multiple cues using a data-driven Markov chain Monte Carlo process," Robotics and Autonomous Systems, vol. 59(11), pp. 966-976, 2011. 
[25] M. Hentschel, et al., "An Adaptive Memory Model for Long-Term Navigation of Autonomous Mobile Robots," Journal of Robotics, vol. 2011, pp., 2012.

[26] B. Bacca, et al., "Appearance-based mapping and localization for mobile robots using a feature stability histogram," Robotics and Autonomous Systems, vol. 59(10), pp. 840-857, 2011.

[27] L. Payá, et al., "Map building and monte carlo localization using global appearance of omnidirectional images," Sensors (Basel, Switzerland), vol. 10(12), pp. 11468, 2010.

[28] F. A. Moreno, et al., "Stereo vision specific models for particle filter-based SLAM," Robotics and Autonomous Systems, vol. 57(9), pp. 955-970, 2009.

[29] S. S. Ge, "Autonomous mobile robots: sensing, control, decision making and applications: CRC press; 2010.

[30] M. Bosse, et al., "An Atlas framework for scalable mapping," in Robotics and Automation, 2003 Proceedings ICRA'03 IEEE International Conference on; 2003: IEEE, Year, pp. 1899-1906.

[31] F. M. Campos, et al., "Global localization with non-quantized local image features," Robotics and Autonomous Systems, vol. 60(8), pp. 1011-1020, 2012.

[32] J. Vallet, et al., "Simultaneous RSS-based localization and model calibration in wireless networks with a mobile robot," Procedia Computer Science, vol. 10, pp. 1106-1113, 2012.

[33] M. Atia, et al., "Particle-Filter-Based WiFi-Aided Reduced Inertial Sensors Navigation System for Indoor and GPS-Denied Environments," International Journal of Navigation and Observation, vol. 2012, pp., 2012.

[34] J. M. Alonso, et al., "Enhanced WiFi localization system based on Soft Computing techniques to deal with smallscale variations in wireless sensors," Applied Soft Computing, vol. 11(8), pp. 4677-4691, 2011.

[35] G. A. Hollinger, et al., "Target tracking without line of sight using range from radio," Autonomous Robots, vol. 32(1), pp. 1-14, 2012.

[36] J. Zhou, et al., "RFID localization algorithms and applications - a review," Journal of Intelligent Manufacturing, vol. 20(6), pp. 695-707, 2009.

[37] G. G. Rigatos, "Extended Kalman and Particle Filtering for sensor fusion in motion control of mobile robots," Mathematics and computers in simulation, vol. 81(3), pp. 590-607, 2010.

[38] B. Cao, et al., "Improved particle filter based on fine resampling algorithm," The Journal of China Universities of Posts and Telecommunications, vol. 19(2), pp. 100-115, 2012.

\section{BIOGRAPHIES OF AUTHORS}
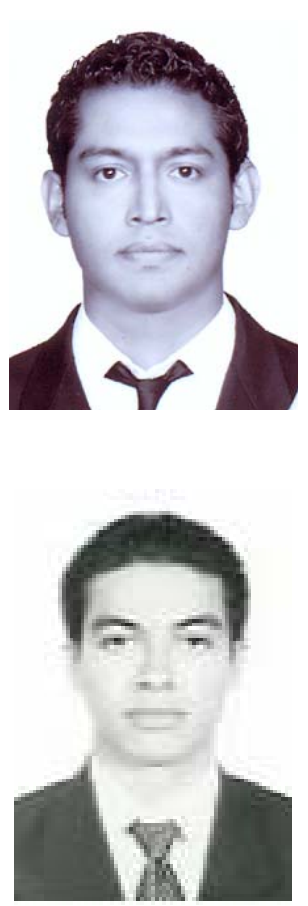

Salvador M. Malagon-Soldara is studying his Ph.D. in the Universidad Autonoma de Queretaro. He has completed his M.Sc. in instrumentation and automatic control in the same university. Terminal lines of Salvador are artificial intelligence, robot control and embedded systems.

Edgar A. Rivas-Araiza received his M. of Sc. degree in Automatic Control from the University of Querétaro and his Ph.D. from the same institution in 2007. His research interests include signal processing, computer vision and motion control. 


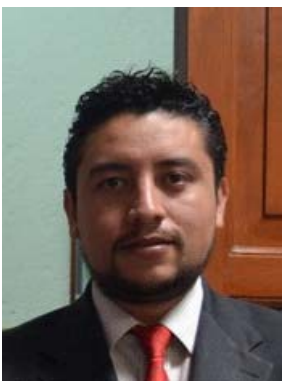

Manuel Toledano-Ayala received his M. of Sc. degree in Automatic Control from the University of Querétaro and his Ph.D in 2010. His research interests include signal processing, telecomunications, renewable energy, and biosystems.

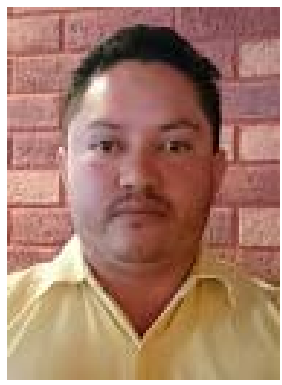

Genaro Soto-Zarazúa received his M. of Sc. degree in Automatic Control from the University of Querétaro and his Ph.D. from the same institution in 2010. His research interests include signal processing, automation, and biosystems.

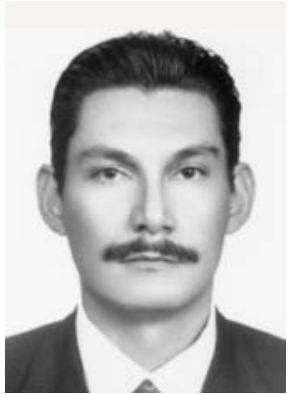

Roberto V. Carrillo-Serrano received his engineering in instrumentation and process control bachelor degree, instrumentation and automatic control master degree, and doctor in engineering degree by the Universidad Autónoma de Querétaro in 2000, 2008, and 2011, respectively. Roberto worked in Kellogg de México from 1999 to 2006. His research areas are robot manipulators control, electric machines control, and underactuated mechanical systems control. Nowadays, Roberto is professor in Universidad Autónoma de Querétaro and he is member of the SNI (national system of researchers) in México. His publications are indexed in JRC database of ISI-Thomson. 